\title{
Activités
}

18-1 | 2021

Le programme de recherche cours d'action (2)

\section{"La construction des parcours de travail en santé et en compétences. Le rôle des régulateurs dans la médiation des parcours des personnels au sol d'une compagnie aérienne" [Résumé]}

Lucie Reboul

\section{OpenEdition}

Journals

Édition électronique

URL : http://journals.openedition.org/activites/6371

DOI : 10.4000/activites.6371

ISSN : 1765-2723

Éditeur

ARPACT - Association Recherches et Pratiques sur les ACTivités

Référence électronique

Lucie Reboul, «"La construction des parcours de travail en santé et en compétences. Le rôle des régulateurs dans la médiation des parcours des personnels au sol d'une compagnie aérienne" [Résumé] », Activités [En ligne], 18-1 | 2021, mis en ligne le 15 avril 2021, consulté le 17 avril 2021. URL : http://journals.openedition.org/activites/6371; DOI : https://doi.org/10.4000/activites.6371

Ce document a été généré automatiquement le 17 avril 2021

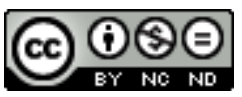

Activités est mis à disposition selon les termes de la licence Creative Commons Attribution - Pas d'Utilisation Commerciale - Pas de Modification 4.0 International. 


\section{"La construction des parcours} de travail en santé et en compétences. Le rôle des régulateurs dans la médiation des parcours des personnels au sol d'une compagnie aérienne" [Résumé]

Lucie Reboul

\section{RÉFÉRENCE}

Lucie Reboul. (2020) La construction des parcours de travail en santé et en compétences. Le rôle des régulateurs dans la médiation des parcours des personnels au sol d'une compagnie aérienne. Thèse de doctorat en Ergonomie. Dirigée par Corinne Gaudart et Catherine Delgoulet. Soutenue le 07/07/20 au CNAM, Paris, École doctorale Abbé Grégoire

\section{NOTE DE L'ÉDITEUR}

\section{Jury de thèse}

M. Pascal UGHETTO, Professeur en sociologie, LATTS, Université Gustave Eiffel (Président)

M. Julien CEGARRA, Professeur en psychologie, ScoTE, INU Champollion (Rapporteur) M. Alain GARRIGOU, Professeur en ergonomie, EPICENE, Université de Bordeaux (Rapporteur) 
Mme Flore BARCELLINI, Professeure en ergonomie, CRTD, CNAM (Examinatrice)

M. Francisco DUARTE, Professeur en ergonomie, COPPE, Université Fédérale de Rio de

Janeiro (Examinateur)

Mme Sonia SUTTER, Ergonome coordinatrice, Air France (Invitée)

\section{Contexte de la recherche : Les parcours de travail des personnels au sol à l'épreuve des transformations du travail}

1 Cette thèse vise à rendre compte des processus de construction et/ou d'usure des parcours de travail des agents (agent de service au client et bagagiste ${ }^{1}$ ) d'une compagnie aérienne, et porte plus précisément sur le rôle de l'activité de travail des régulateurs que nous qualifions ici de "médiatrice». Elle s'inscrit dans une démarche de recherche-action (Delgoulet, Gaudart, Molinié, Volkoff, Cabon, Reboul et al., 2020) et vise le développement d'une prévention durable et collective de la santé à travers le projet de systèmes de travail et de parcours dits "soutenables" (Shani, Docherty, \& Forslin, 2002; Volkoff \& Gaudart, 2015). La compagnie aérienne en question est marquée par un rythme continu de changements multiples (Bradley, 2000) qui ont des temporalités distinctes et dont la rencontre est porteuse de dyschronies (Alter, 2003; Gaudart, 2014) entre : la digitalisation de la relation de service client des agents de service aux clients, les formes de flexibilités interne et externe des équipes de bagagistes (polyvalence, sous-traitance partielle), la rationalisation des effectifs, et un vieillissement marqué par un arrêt de recrutement. Dans ce contexte, le travail des agents s'intensifie (Gollac \& Volkoff, 1996 ; Green \& Macintosh, 2001), produisant des parcours-usure ${ }^{2}$ (Buchmann, Mardon, Volkoff, \& Archambaud, 2018 ; Gaudart \& Ledoux, 2013 ; Reboul, Delgoulet, Gaudart, \& Sutter, 2020). Toutefois, l'organisation du travail (incluant les horaires de travail, le contenu, la charge et les rythmes du travail, les relations interpersonnelles, les choix d'affectation aux tâches, etc.) des agents est aussi en partie façonnée par les encadrants de premier niveau, appelés dans la compagnie aérienne les « régulateurs ». L'hypothèse était alors que l'activité des régulateurs est potentiellement vectrice de la construction de la santé des agents et contribuerait à des parcours-construction ${ }^{3}$.

\section{Cadre théorique et thèse défendue : Le rôle de l'activité médiatrice des régulateurs}

2 Les régulateurs sont des encadrants informels (Wolff, 2013) qui, dans le cadre de situations d'ordonnancement des tâches sur les plannings des agents (Berglund \& Karltun, 2007 ; Cegarra, 2008), effectuent un travail d'organisation (de Terssac, 2011) et déploient des régulations conjointes (Reynaud, 1988) entre différentes logiques hiérarchiques. En effet, les régulateurs sont pris en étau entre des prescriptions managériales (descendantes) et des prescriptions provenant du travail réel des agents (ascendantes) (Six, 1999). Aussi, ils ont pour missions de préserver la santé des agents (notamment en tenant compte des restrictions médicales) et de développer les compétences tout en ayant pour consigne « d'optimiser les plannings » et de s'assurer de «l'efficacité opérationnelle». Enfin, ils s'assurent de créer des alliances entre des 
temporalités individuelles (les parcours en santé et en compétences de chaque salarié), collectives (les règles de métier, les modalités d'attribution des tâches et de progression de carrière) et gestionnaires (les projets liés à la digitalisation et la flexibilité). Ce travail d'organisation se caractérise par des réajustements permanents des plannings pour gérer les nombreux événements issus de la variabilité de la production (flux de vols et de clients) et des agents (variabilité inter et intra-individuelle). Notre thèse défend l'hypothèse que les régulateurs, au travers de leurs choix d'ordonnancement des tâches, élaborent des pratiques de prévention informelles et "profanes " (Dodier, 1986), c'est-à-dire qui se construisent dans la confrontation quotidienne des situations de travail et se démarquent ainsi de celles des acteurs de santé au travail, empreintes d'une conception médico-légale et normative de la santé. Nous qualifions cette activité de médiatrice à partir de la définition suivante: "servir d'intermédiaire entre deux ou plusieurs choses $»^{4}$. Cette notion donne à voir les différentes contradictions que les régulateurs gèrent au cours de leur prise en charge d'événements, que nous avons regroupées en trois grandes facettes antagoniques:

- Les pôles «santé » et "production », souvent gérés de manière cloisonnée par les services de santé au travail et de production dans les entreprises ;

- Des temporalités multiples, qui peuvent présenter des dyschronies ;

- Des prescriptions descendantes et ascendantes relevant des logiques de la hiérarchie et de celles des agents.

3 L'usage du terme de médiation a aussi pour but de montrer la visée développementale de l'activité des régulateurs, en soulignant les effets à court et long termes que les solutions innovantes et originales créées génèrent sur l'activité et la santé des agents, et en cela, les transforment.

\section{Méthodologie : Combiner des perspectives diachronique et synchronique pour comprendre les relations santé/travail à l'échelle des parcours}

4 Pour étudier le rôle médiateur du travail des régulateurs dans la construction des parcours des agents, nous avons utilisé des outils quantitatifs et qualitatifs, propres à la démographie du travail et à l'ergonomie (Volkoff \& Molinié, 2011) en vue de combiner une perspective diachronique, i.e. les évolutions des conditions de travail et des parcours individuels et collectifs et synchronique, i.e. l'analyse des articulations ou tensions entre les différentes temporalités du point de vue de l'activité de médiation des régulateurs.

5 L'analyse des parcours des personnels au sol ${ }^{5}$ s'est effectuée à partir des bases de données de la compagnie aérienne de manière à retracer les évolutions sociodémographiques (fluctuation des effectifs, vieillissement, états de santé) et technico-organisationnelles. Elles ont été complétées par l'analyse des représentations de ces transformations par 27 acteurs décisionnaires. En parallèle, douze entretiens biographiques ont été réalisés auprès des agents de service au client et des bagagistes dans l'objectif de reconstituer leurs parcours de travail.

6 L'étude des processus de médiation s'est faite à partir de l'analyse de la prise en charge d'événements. Ces événements ont été recueillis au cours de 29 observations systématiques du travail des régulateurs au sein de deux sites (en Île-de-France et en 
région Provence-Alpes-Côte d'Azur) et des deux métiers (agent de service au client et bagagiste). Nous avons caractérisé la gestion des événements selon différentes modalités : le mode de prise en compte (détecté par les régulateurs ou bien «subis", c'est-à-dire faisant l'objet d'une demande extérieure), la typologie (de source santé ou production), les communications impliquées et le mode de gestion.

\section{Résultats : Les caractéristiques de l'activité de médiation}

7 Les résultats instruisent les questions de recherche suivantes: 1) caractériser les processus de médiation: l'ampleur des enjeux de santé et les modes de gestion associés; 2) identifier des ressources qui les soutiennent et en permettent le développement (collectif, expérience); et 3) cibler les contraintes qui les amenuisent ou les empêchent.

8 L'analyse des événements montre que ceux appartenant à la catégorie "santé" représentent entre $20^{6}$ et $30 \%^{7}$ des événements gérés par les régulateurs. Ces événements de santé comprennent les restrictions médicales, mais aussi, et surtout d'autres indicateurs qui relèvent de différentes dimensions de la santé (physique, cognitive, sociale, psychique) : l'infrapathologie (douleurs, plaintes, fatigue, etc.), des compétences, du collectif, des horaires de travail, du contenu du planning et des rythmes de travail, etc. Les stratégies de médiation consistent notamment à créer des solutions pour tenir compte des impératifs de production et des enjeux de santé, et de cette manière favoriser des situations de travail soutenables pour les agents. Elles résolvent les dyschronies entre les temporalités gestionnaires, qui cherchent principalement à « optimiser les plannings » pour gagner en ressource, les temporalités individuelles (les particularités en santé et en compétences des agents) et les temporalités collectives (les logiques d'équité dans l'attribution des tâches). Enfin, elles permettent la rencontre entre la prescription managériale (satisfaction client, quantité, délais, sécurité) et le travail réel (la variabilité, les aléas) des agents. Les médiations favorisent aussi une meilleure gestion de la charge de travail des régulateurs, car l'anticipation des configurations à risque, à court et à long termes, leur donne de la marge de manœuvre (réguler la survenue d'événements "subis ", disposer davantage de flexibilité en montant en compétences les agents et en évitant leur usure accélérée, etc.). Ainsi, quand bien même les missions des régulateurs sont essentiellement axées sur des objectifs productifs, ces résultats montrent que leur travail est en partie dédié à la gestion et à la prévention de la survenue de problèmes de santé. En cela, cette activité médiatrice contribue à des parcours-construction.

Loin d'être un travail individuel, les résultats indiquent que 88 \% des événements sont gérés de manière collective: d'une part avec les collègues lorsque les régulateurs travaillent en équipe, et d'autre part avec les agents. Les régulations collectives avec les régulateurs (résolution collective de problème, transmission d'informations, entraide, etc.) témoignent de l'existence d'un "référentiel opératif commun " (Leplat, 1991) portant sur les caractéristiques des agents et des tâches et se basant sur des valeurs partagées par rapport aux conditions de travail des agents et la préservation de leur santé. Ces échanges permettent d'actualiser et d'enrichir les connaissances de chaque régulateur et donc de développer les médiations possibles. La fréquence et le contenu des échanges entre agents et régulateurs montrent que l'efficacité des médiations 
repose aussi sur des coopérations informelles régies par le principe du «donnantdonnant». Dans le cadre d'une activité fortement marquée par de la réactivité (la plupart des événements sont «subis»), les résultats montrent que les régulateurs parviennent tout de même à détecter $20 \%$ d'événements de source «santé » (vs $30 \%$ d'événements de source "production »). Pour détecter des configurations à risque et éviter que celles-ci se répercutent directement sur l'activité des agents, les régulateurs mobilisent leur expérience et leurs propres connaissances en matière de liens santé/ travail. Ces connaissances, qui diffèrent des définitions médico-légales, sont le fruit d'une redéfinition des pénibilités en fonction des situations de travail qu'ils ont vécues en tant que régulateurs, mais aussi en tant qu'anciens agents. Les régulateurs ont donc élaboré leurs propres indicateurs de gestion de la santé et des compétences de leurs équipes, cependant ces médiations sont moins du fait d'un choix ou d'un tempérament que d'une contrainte donnée. En effet, il n'existe pas de fortes disparités chez les régulateurs ni en fréquence d'événements de source "santé », ni dans leurs modes de gestion.

10 Enfin, les résultats identifient plusieurs configurations de contraintes qui peuvent empêcher la mise en œuvre d'une activité de médiation et en cela participer à la fabrication de parcours-usure. En effet, l'efficacité des médiations repose sur les possibilités qu'ont les régulateurs de détecter des anomalies ou des améliorations à apporter sur les plannings. Or, ces possibilités de détection diminuent d'un peu moins de dix points avec la densification de leur travail ${ }^{8}$. Cela est associé à des changements de plannings dans le cadre de décisions plus rapidement prises, des omissions et des arbitrages au détriment des aspects de la santé. Cela donne lieu à des plannings incohérents dans leur enchaînement spatio-temporel, des recouvrements de tâches, des suppressions de temps non directement productifs (formation, pause, etc.) et de certains critères décisionnels (caractéristiques des agents, des plannings précédents, etc.). Ces médiations dépendent aussi des dimensionnements en effectif (nombre d'agents disponibles selon leurs spécificités en compétences et santé) et de la typologie des tâches. Enfin, la localisation de la régulation dans les structures de l'aéroport (dans les mêmes locaux que les agents ou dans un bâtiment à part) joue aussi sur les possibilités d'élaborer des médiations. Cet ensemble de configurations de contraintes peuvent alors empêcher l'élaboration de médiations et se répercuter sur l'activité et la santé des agents, en produisant de l'usure par une intensification du travail: optimisation des plannings, suppression des temps non directement productifs (formation, pause, réunion d'équipe, récupération) et usage immédiat des compétences au détriment de leur développement.

\section{Conclusion/discussion : Développer l'activité de médiation}

11 Cette thèse permet de révéler une activité de médiation multifacettes déployée par les régulateurs. Elle permet une prévention collective et durable des risques, et participe ainsi à fabriquer des parcours-construction. Cette activité se traduit par une gestion permanente d'événements dont une part importante est de source "santé », que nous appréhendons ici de manière holistique et du point de vue du travail des régulateurs. $\mathrm{Si}$ ces dimensions demeurent non prises en compte par les conceptions des organisations du travail, l'analyse de leur activité montre qu'elles sont malgré tout intégrées dans un 
"travail d'organisation " afin de s'assurer du bon fonctionnement du service et du métier. Ces résultats montrent ainsi le rôle décisif, mais cependant invisible du travail des encadrants de premier niveau : ceux-ci résolvent des contradictions non pensées en amont au croisement de la gestion des ressources humaines, de la prévention de la santé et d'objectifs de production. Cette vision du travail des régulateurs amène alors à considérer les ressorts de cette activité en vue de la développer, notamment en favorisant les régulations collectives (avec les agents et avec les régulateurs) et en mettant au jour leurs compétences tacites. Ces médiations reposent sur l'expérience d'événements vécus, qui s'élabore dans un espace de médiation. Cet espace symbolique est constitué d'un ensemble de combinaisons possibles qui offre en situation une palette de choix de médiation. Ces combinaisons produisent alors des «espaces intermédiaires » entre le passé et le présent des parcours individuels et collectifs, ainsi qu'entre la santé et la production. Plusieurs pistes de réflexion émergent pour soutenir et développer cette activité de médiation, au niveau de la charge de travail, des configurations des postes et des équipes, de l'organisation du travail des agents et de la conception des parcours de travail.

\section{BIBLIOGRAPHIE}

Alter, N. (2003). Mouvement et dyschronies dans les organisations. L'Année sociologique, 53(2), 489-514.

Berglund, M., \& Karltun, J. (2007). Human, technological and organizational aspects influencing the production scheduling process. International Journal of Production Economics, 110, 160-174 Bradley, G. (2000). The information and communication society: how people will live and work in the new millennium. Ergonomics, 43(7), 844-857.

Buchmann, W., Mardon, C., Volkoff, S., \& Archambaud, C. (2018). Peut-on élaborer une approche ergonomique du « temps long »? Une étude des douleurs articulaires liées au travail, dans une grande entreprise. PISTES, 20-1. DOI : 10.4000/pistes.5565.

Cegarra, J. (2008). A cognitive typology of scheduling situations: a contribution to laboratory and field studies. Theoretical Issues in Ergonomics Science, 9(3), 201-222.

Delgoulet, C., Gaudart, C., Molinié, A.-F., Volkoff, S., Cabon, P., Reboul, L., Cuvelier, L., \& Toupin, C. (2020). Des fragilités individuelles aux processus de fragilisation au travail. La revue des conditions de travail, $\mathrm{n}^{\circ} 11$.

Dodier, N. (1986). Corps fragiles. La construction sociale des événements corporels dans les activités quotidiennes du travail. Revue française de sociologie, 27(4), 603-628.

Gaudart, C. (2014). Les relations entre l'âge et le travail comme problème temporel. PISTES, 16-1.

Gaudart, C., \& Ledoux, E. (2013). Parcours de travail et développement. In P. Falzon (Ed.), Ergonomie constructive (pp. 117-132). Paris : PUF.

Gollac, M., \& Volkoff, S. (1996). Ciltius, altius, fortius (l'intensification du travail). Actes de la recherche en sciences sociales, 114, 54-67. 
Green, F., \& Macintosh S. (2001). The intensification of work in Europe. Labour Economics, 8(2), 291-308.

Leplat, J. (1991). Activités collectives et nouvelles technologies. Le Travail Humain, 43(1), 117-223.

Reboul, L., Delgoulet, C., Gaudart, C., \& Sutter, S. (2020). La digitalisation de la relation de service : conséquences sur la santé et sur les parcours des agents de services au client d'une compagnie aérienne. PISTES, 22-1. DOI : https://doi.org/10.4000/pistes.6851.

Reynaud, J.-D. (1988). Les régulations dans les organisations : régulation de contrôle et régulation autonome. Revue française de sociologie, 29, 5-18.

Six, F. (1999). De la prescription à la préparation du travail. Apports de l'ergonomie à la prévention et à l'organisation du travail sur les chantiers du BTP. Mémoire HDR, Université Charles de GaulleLille 3.

Shani, A.-B., Docherty, P., \& Forslin, J. (2002). Creating Sustainable Work Systems - Emerging Perspectives and Practices. London : Routledge.

Terssac (de), G. (2011). Théorie du travail d'organisation. In B. Maggi (Ed.) Interpréter l'agir : un défi théorique (pp. 97-121). France : PUF.

Volkoff, S., \& Gaudart, C. (2015). Working conditions and "sustainability": Converting Knowledge into Action. Centre d'études de l'emploi, Rapport de recherche, $\mathrm{n}^{\circ} 92$.

Volkoff, S., \& Molinié, A.F. (2011). L'écheveau des liens santé-travail, et le fil de l'âge. In A. Degenne, C. Marry, \& S. Moulin (Eds.), Les catégories sociales et leurs frontières (pp. 323-344). Laval (Qc) : Presses de l'Université.

Wolff, L. (2013). Un encadrement de plus en plus... encadrés. Santé et Travail, nº 82, 30-32.

\section{NOTES}

1. Les agents de service aux clients sont en charge de l'accueil, l'enregistrement et l'embarquement des clients. Les bagagistes ont pour mission l'acheminement, chargement et déchargement des bagages, et les opérations liées à l'accueil et au départ des avions.

2. Des parcours produisant de la déqualification, des restrictions médicales, de la relégation voire de l'exclusion et de la sortie de l'emploi.

3. Des parcours sources de construction de la santé au moyen du développement des compétences et de mise à l'abri des contraintes.

4. https://www.cnrtl.fr/definition/médiation

5. Selon deux grandes directions : parcours-usure vs parcours-construction.

6. Métiers du pôle Client (agent de service client).

7. Métiers du pôle Avion (bagagiste).

8. La densité correspond au nombre d'événement par tranche horaire. Elle peut être modérée, moyenne ou forte. 
INDEX

Mots-clés : parcours de travail, encadrant de premier niveau, ordonnancement des tâches, activité de médiation, échelles de court et long termes, santé et performance, prescriptions descendantes et ascendantes, temporalités 